\title{
Correlating Local Chemistry and Local Cation Displacements in the Relaxor Ferroelectric PMN
}

\author{
Matthew J. Cabral ${ }^{1}$, Shujun Zhang ${ }^{2}$, Jocelyn $\mathrm{Chi}^{3}$, Brian J. Reich ${ }^{3}$, Elizabeth C. Dickey ${ }^{1}$ and James M. \\ LeBeau $^{1}$ \\ 1. Department of Materials Science \& Engineering, North Carolina State University, Raleigh NC. \\ 2. Institute for Superconducting and Electronic Materials, Australian Institute of Innovative Materials, \\ University of Wollongong, Australia \\ 3. Department of Statistics, North Carolina State University, Raleigh NC.
}

Relaxor ferroelectrics are a unique class of materials that can be identified by their high dielectric constants, low hysteresis, large electrostrictive strains, and diffuse paraelectric to ferroelectric phase transitions [1]. The outstanding electromechanical response of relaxor ferroelectrics is attributed to the presence of "polar nanoregions" (PNRs), nanoscale domains that coexist within normal ferroelectric domains. Although relaxors have been extensively studied, the structural and chemical origins of PNRs are not yet fully understood.

In this talk, we will present an investigation of local distortion and its relationship with local chemistry in the prototypical relaxor, $\mathrm{Pb}\left(\mathrm{Mg}_{1 / 3} \mathrm{Nb}_{2 / 3}\right) \mathrm{O}_{3}(\mathrm{PMN})$. In this perovskite structured material, the $\mathrm{A}$ sublattice contains $\mathrm{Pb}$, while the $\mathrm{B}$ sub-lattice contains $\mathrm{Mg} / \mathrm{Nb}$ at a 1:2 ratio. In addition to PNRs, chemical ordering has also been observed in PMN in the form of chemically ordered regions (CORs) which are $\sim 2-5 \mathrm{~nm}$ in size. CORs can be characterized by a charge balanced "random site" model that assumes a $1: 1 \mathrm{~Pb}\left(\mathrm{~B}_{1 / 2}^{\mathrm{I}} \mathrm{B}_{1 / 2}^{\mathrm{II}}\right) \mathrm{O}_{3}$ ordered structure where $\mathrm{B}^{\mathrm{I}}$ is a random mixture of $\left(\mathrm{Mg}_{2 / 3} \mathrm{Nb}_{1 / 3}\right)$ and $\mathrm{B}^{\mathrm{II}}$ contains only $\mathrm{Nb}[2]$. Through the application of annular dark-field scanning transmission electron microscopy (ADF-STEM) in figure $1 \mathrm{a}$, we identify distinct $\mathrm{B}^{\mathrm{I}}$ and $\mathrm{B}^{\mathrm{II}}$ positions across the image, as in figure $1 \mathrm{~b}$. As is evident, $\mathrm{B}^{\mathrm{I}}$ (blue circles) and $\mathrm{B}^{\mathrm{II}}$ (red circles) positions can be identified and classified into unique domains across the entire image, separated by antiphase boundaries (green circles). Further, we will discuss and justify the development of an "ordering metric", here defined as the standard deviation of local B-site intensities. Figure 1c highlights how highly ordered regions transition diffusely to less order. Minimum values of local ordering correspond to antiphase regions. These results are also discussed in the context of multislice STEM image simulations, where the effect of chemical fluctuations on the ordering metric is determined [3].

The relationship between chemical ordering and local distortion is elucidated through the application of spatial statistics. Assuming a rigid B-sub lattice, we determine local A-site distortions as shown in figure 2a. The application of revolving STEM (RevSTEM) enables picometer precision when measuring distortions [4]. The square roots of the A-site distortions are then correlated with the local means and local standard deviations of the B-site intensities. Utilizing multiple linear regression and a Bayesian fit of a spatial linear model, non-random correlation between distortion and intensity is evident. Spatial correlation between A-site distortion and B-site intensity is then found to be $\sim 1.5-2.0$ $\mathrm{nm}$, the expected size of a PNR (figure 2b). Finally, we will discuss the broad applications of these techniques and implications in the wider relaxor ferroelectric community [5]. 


\section{References:}

[1] S. Zhang and F. Li, Journal of Applied Physics 111, (2012), 031301.

[2] P.K. Davies and M.A. Akbas, Journal of Physics and Chemistry of Solids 61 (2000).

[3] E.J. Kirkland et al, Ultramicroscopy 23 (1987).

[4] X. Sang and J.M. LeBeau, Ultramicroscopy 138 (2014).

[5] This material is based upon work supported by the National Science Foundation, as part of the Center for Dielectrics and Piezoelectrics under Grant Nos. IIP-1361571 and IIP-1361503. The authors acknowledge the use of the Analytical Instrumentation Facility at NCSU, which is supported by the state of North Carolina and the National Science Foundation. M.J.C acknowledges support from the National Science Foundation, as part of NRT-SEAS under Grant No, DGE-1633587
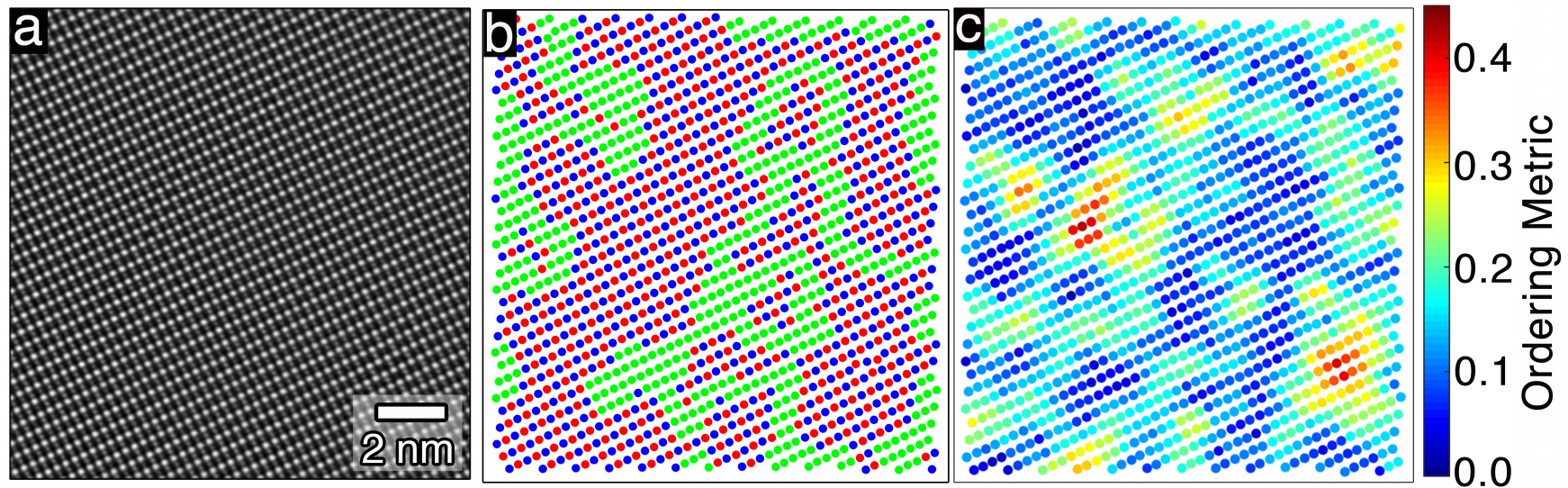

Figure 1. (a) ADF-STEM image of $<011>$ oriented PMN. (b) $B^{I}$ (blue) and $B^{I I}$ positions (red) separated by antiphase boundaries (green). (c) Ordering metric values determined across the image.
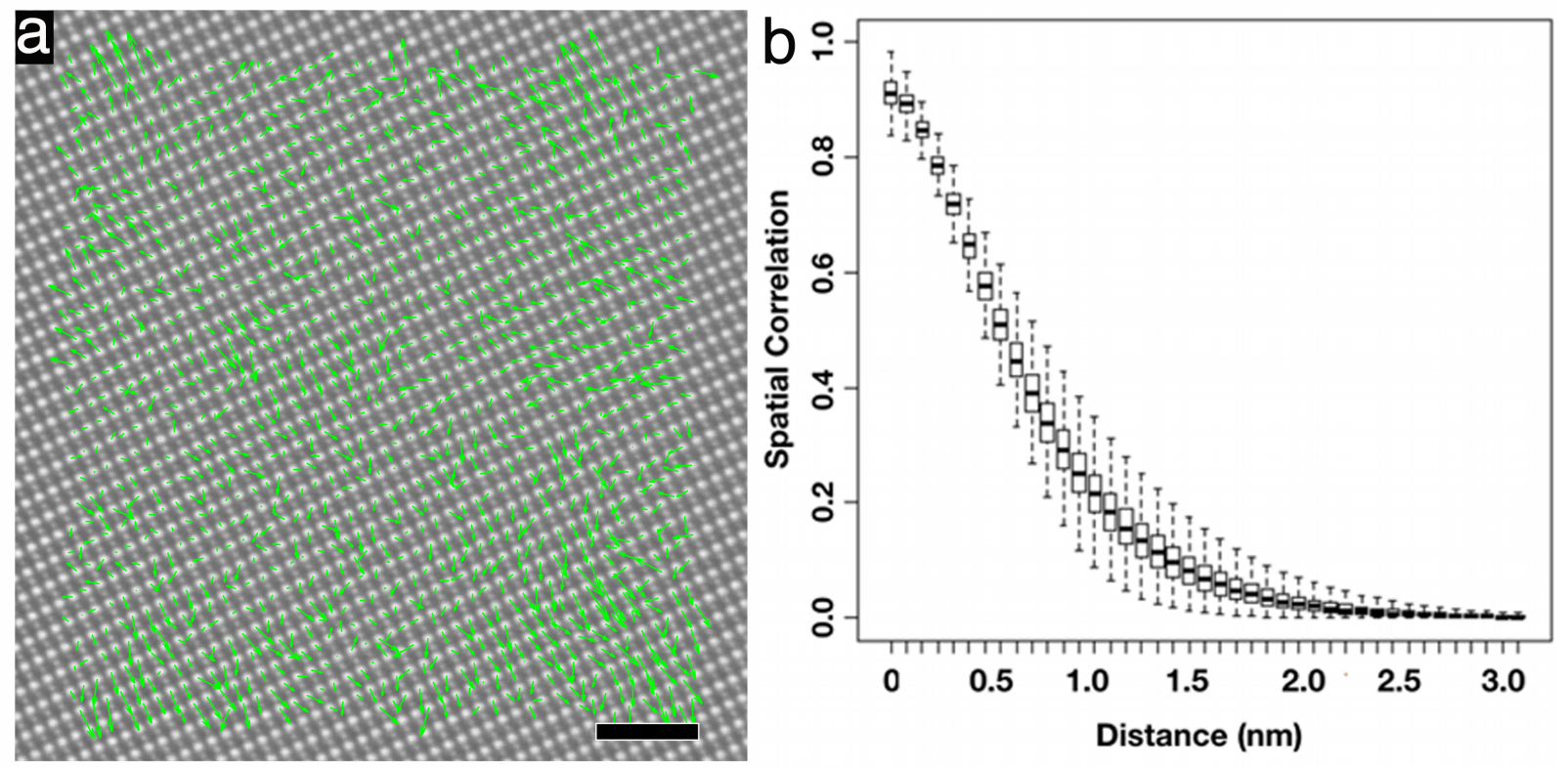

Figure 2. (a) Distortion map indicating direction of A-site displacement. (b) Spatial correlation vs. distance for PMN. Scale bar represents $2 \mathrm{~nm}$. 